\title{
Strates
}

STRATES Matériaux pour la recherche en sciences sociales

$4 \mid 1989$

Dossier : images réfléchies. Paroles d'un paysan révolutionnaire

\section{Paroles de Bernard Lambert : un paysan révolutionnaire}

Bernard Lambert, Françoise BOURQUELOT et Nicole Mathieu

\section{(2) OpenEdition \\ Journals}

Édition électronique

URL : http://journals.openedition.org/strates/4732

DOI : $10.4000 /$ strates.4732

ISSN : $1777-5442$

Éditeur

Laboratoire Ladyss

Édition imprimée

Date de publication : 31 décembre 1989

ISSN : 0768-8067

Référence électronique

Bernard Lambert, Françoise BOURQUELOT et Nicole Mathieu, «Paroles de Bernard Lambert : un paysan révolutionnaire », Strates [En ligne], 4 | 1989, mis en ligne le 19 mai 2008, consulté le 07 septembre 2020. URL : http://journals.openedition.org/strates/4732 ; DOI : https://doi.org/10.4000/ strates. 4732

Ce document a été généré automatiquement le 7 septembre 2020

Tous droits réservés 


\title{
Paroles de Bernard Lambert : un paysan révolutionnaire
}

\author{
Bernard Lambert, Françoise BOURQUELOT et Nicole Mathieu
}

1 Le texte que nous publions est la transcription fidèle des paroles prononcées par Bernard Lambert le 4 mai 1981 au séminaire «Luttes dans et pour l'espace rural» de Françoise Bourquelot et Nicole Mathieu à l'Ecole des Hautes Etudes en Sciences Sociales (EHESS).

2 Il s'inscrit dans une ambition : donner une chance à la gauche paysanne d'exister dans l'histoire du temps présent, sans a priori, sans thèse préconçue et en laissant d'abord le choix de la réflexion avant l'interprétation et l'enfermement qu'elle induit.

3 Il existe pour l'instant peu de textes sur l'histoire de la gauche paysanne telle qu'elle s'est manifestée après 1968. Ce sont encore des matériaux (journaux et revues dont certaines aujourd'hui disparues, monographies de luttes diverses et dispersées, livres de militants...) qui permettraient de la retracer. Déjà pourtant plusieurs thèses s'opposent qui se sont exprimées lors du colloque de l'Association Française de Science Politique Les agriculteurs et la politique depuis 1970. On peut les résumer en trois positions.

4 La première, mettant en avant l'analyse institutionnelle, définit la gauche paysanne comme «le produit contradictoire et toujours mouvant d'une dépendance organique des syndicats au Ministère de l'Agriculture, d'une lutte pour la représentativité et la pluralité syndicale, de l'émergence de professionnels-syndicaux»; pessimiste, elle insiste sur "l'écart entre le discours idéologique 》 (par exemple cet «idéologue charismatique, B. Lambert») «et le traitement effectif des dossiers qui s'accroît au fur et à mesure que les syndicats s'institutionnalisent». "Entre dissidence et concurrence» la seule issue est l' "institutionnalisation", les vrais enjeux sont de "nouer des alliances pour les échéances électorales» (Lagrave, 1987). Position de recherche qui conclut "la nouvelle gauche syndicale est parvenue à créer sinon une alternative syndicale, à tout le moins un contre-poids et un contre-pouvoir au syndicalisme dominant » (Hubscher, Lagrave, 1989).

5 La deuxième, régionaliste, fait de l'Ouest le pôle fondateur à partir duquel s'est diffusée la pensée de la «nouvelle gauche paysanne ». Mais elle n'est pas une thèse au même titre que la première, car elle provient de ceux-là même qui font l'histoire. Car si elle 
attribue «les origines idéologiques de la nouvelle gauche paysanne» au "mouvement paysan de Loire-Atlantique ", ou plus généralement aux paysans de l'Ouest, c'est parce que ses auteurs ont été des acteurs de cette histoire (Bourrigaud, 1987). Il faut d'ailleurs admettre que la paysannerie de l'Ouest est restée une paysannerie nombreuse où la question de l'organisation de minorités est encore soluble et où la visibilité des actions est donc plus éclatante.

6 La troisième, la nôtre, met en avant la notion de rupture et tente de «discuter et de vérifier que les années 1968-1974 sont bien une période où s'effectue un renversement idéologique dans une fraction minoritaire de la paysannerie » (Bourquelot, Mathieu, 1987), qui conduit à des remises en cause radicales, mais surtout à de nouvelles pratiques sans rapport avec les institutions syndicales. Mais au-delà de cette position qui ressemble plus à une hypothèse, tant est grande la multiplicité des mouvements et des lieux qui ont contribué à cette rupture, nous avons choisi de donner d'abord la parole à ceux dont c'est l'histoire, de ne pas enfermer trop vite l'histoire de la gauche paysanne dans les querelles d'interprétation: une déviation de la modernisation? un avatar du gauchisme? un rejeu d'une pensée de gauche traditionnelle dans la paysannerie ?... Pour commencer, la parole est à Bernard Lambert, c'est notre manière de rendre hommage à la Révolution française.

7 Bernard Lambert (1931-1984) ${ }^{1}$ est une figure politique qui a marqué le syndicalisme paysan par sa constante capacité de contestation et de rupture. Bernard Thareau écrit dans la préface du livre récemment paru Bernard Lambert, trente ans de combat paysan: "Bernard était un fonceur, un apporteur d'idées, toujours en éveil sur les transformations du monde paysan et de la société rurale. Impatient d'être l'acteur, pressé d'être l'orateur et le porte-parole de la lutte des paysans pour plus de dignité, des petits pour plus de justice."

8 Il est aussi bien connu des chercheurs en sciences sociales qui ont à faire avec l'agriculture, ne serait-ce que parce qu'il a publié en 1970 un livre Les paysans dans la lutte des classes, qui répondait dix ans après à celui de Michel Debatisse La révolution silencieuse. Mais l'est-il suffisamment en dehors de ce cercle et là où, par une tradition qui remonte à Hegel, paysannerie est synonyme de conservatisme, de réaction et de contre-révolution.

Or Bernard Lambert est un personnage que l'on peut qualifier de « révolutionnaire ». Se servir de la parole pour convaincre, mêler toujours la réflexion à l'action, remettre incessamment en cause ce qui paraît établi, y compris la religion et ce que l'on a établi soi-même, inventer les formes d'action si elles servent la justice, refuser le corporatisme et privilégier le collectif, penser la paysannerie comme la société traversées par la lutte des classes, combattre donc en liant sa lutte à celle des ouvriers et des exploités... tels sont les éléments du portrait qui fondent le qualificatif. Avec sa belle personnalité, il n'est pourtant pas exceptionnel, ou plutôt il l'est, comme et avec beaucoup d'autres militants de mouvements minoritaires dont on ne connaît pas les noms et qu'il a su illustrer.

10 Ce texte doit être replacé dans son contexte: quelques jours avant l'arrivée de la gauche au pouvoir et donc avant le regroupement de tous les mouvements paysans minoritaires dans le syndicat CNSTP. On y trouvera, outre l'intérêt de découvrir la saveur de ce personnage haut en couleurs, ${ }^{2}$ le récit de trente ans d'histoire du syndicalisme paysan depuis ses origines dans la JAC. Mais cette histoire est toute entière tournée vers l'explication de la naissance et de l'évolution du mouvement des 
«Paysans travailleurs" dont Bernard Lambert fut un des fondateurs. Ce texte est parcouru par les débats de l'époque, dont certains sont très datés, mais il montre encore ce qu'est une pensée d'avant-garde, la force de l'ouverture sur les problèmes internationaux comme sur les autres catégories de travailleurs, la capacité de se projeter dans l'avenir et d'agir. Nous avons présenté ce texte de manière à faire ressortir les hypothèses sur les formes principales de ruptures qui caractérisent cette gauche paysanne. ${ }^{3}$

Françoise Bourquelot et Nicole Mathieu

F. BOURQUELOT , N. MATHIEU, 1987, « Aux origines des ruptures paysannes : les

années 1968 à 1974 ", in Les agriculteurs et la politique depuis 1970, Colloque des 30 novembre, 1 et 2 décembre 1987, Association Française de Science Politique, Paris.

R. BOURRIGAUD, 1987, « Origines idéologiques de la nouvelle gauche paysanne : le cas de

la Loire-Atlantique ", in Les agriculteurs et la politique..., op. cit.

Y. CHAVAGNE, 1988, Bernard Lambert, Trente ans de combat paysan. Préface de Bernard Thareau, La Digitale/Association Bernard Lambert, Quimperlé.

M. DEBATISSE, 1963, La révolution silencieuse, Calmann-Lévy, Paris.

R. HUBSCHER, R.M. LAGRAVE, 1989, France : une fraternité en lutte », in « Les organisations syndicales et professionnelles agricoles en Europe », Cahiers du CEVIPOF, 5, Paris.

R.M. LAGRAVE, 1987, « La gauche paysanne : entre dissidence et concurrence (MODEF,

CNSTP, FNSP) ", in Les agriculteurs et la politique....op. cit.

B. LAMBERT, 1970, Les paysans dans la lutte des classes, Le Seuil, Paris.

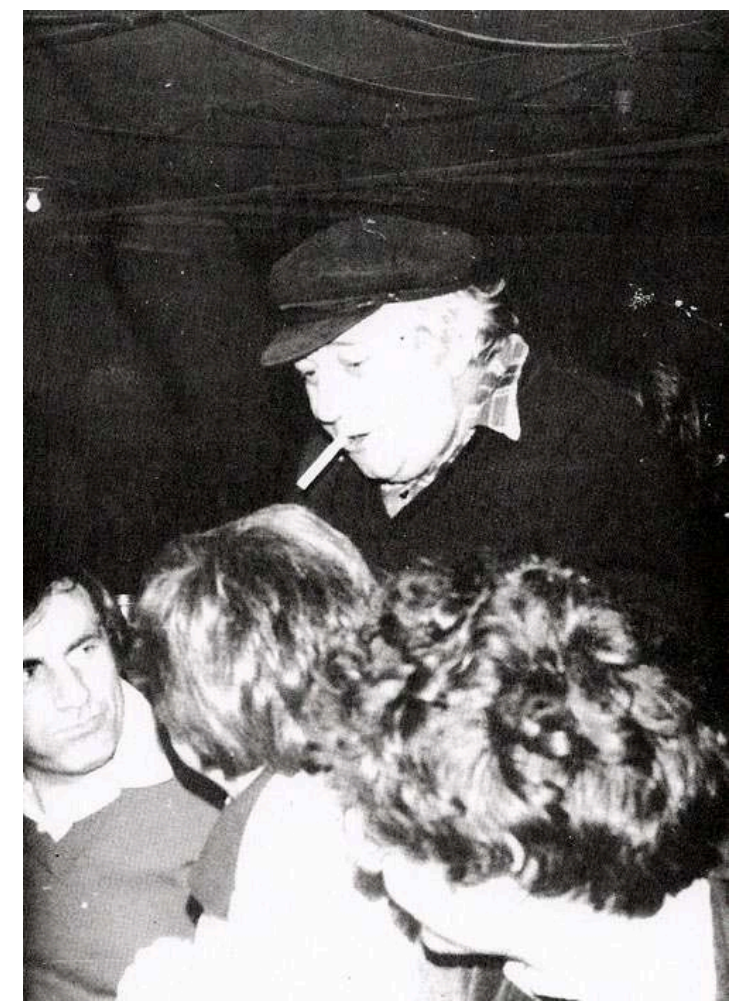

1984, Bernard Lambert avec sa casquette, dans une fête privée rassemblant les militants paysans. 
11 Pour comprendre la manière dont s'est fait l'accouchement de « Paysans travailleurs ", il est nécessaire, très brièvement, de rappeler ce qui à mon sens a été décisif quant à la recherche nouvelle, à la remise en cause du ronron habituel.

12 Ce n'est pas un secret de dire qu'une autre expression syndicale, ou le besoin d'une autre forme d'action syndicale, a pris naissance principalement dans une région de France - l'Ouest - à partir des années soixante, pour des raisons très simples. On était dans une zone où l'industrialisation ne se faisait pas - il y avait de grosses batailles sur le désenclavement de la Bretagne - et où les industries classiques connaissaient déjà une première vague de crise - les chantiers navals, le peu de sidérurgie qu'il pouvait y avoir (les Forges d'Hennebont), la chaussure, le textile... C'était une région exportatrice de main d'œuvre, la démographie paysanne était relativement forte, principalement pour des raisons culturelles, autant dire religieuses. L'industrialisation de l'agriculture - les techniques permettant qu'elle soit progressivement mise en place - pouvait se faire avec davantage d'espérance de profit dans cette région plutôt que dans une autre.

Ce n'est pas par hasard si UNILEVER, avec la COFNA ${ }^{4}$ et RALSTOM avec DUQUESNEPURINA, CARG1L, SANDERS, puis les grands groupes coopératifs, dans la foulée, ainsi que quelques gros commerçants du coin, assez gros minotiers - du type GUYOMARCH qui rapidement est devenu autre chose qu'un individu, puisque la Banque R0TSCH1LD et le Crédit Agricole en sont partie prenante -, le MELINER, ont commencé à mettre en place de nouveaux systèmes de production très concentrés, des élevages, alimentés de l'extérieur, en hors sol. Et cela a bien sûr commencé dans la volaille, avec la mise en place d'intégrations très unilatérales et très sauvages à l'époque. Ils l'ont fait parce qu'on était une région-pilote pour usines-pirates - les usines qui piratent la maind'œuvre. Compte tenu des problèmes de l'emploi, à partir du moment où on promettait à un jeune ou à un paysan qu'il pourrait se maintenir et qu'il ne serait pas condamné à foutre le camp - même si les promesses n'étaient pas matérialisées, si c'était des promesses verbales -, la tentation était forte de tout faire plutôt que de partir. Ils ne sont pas venus parce qu'ils nous aimaient bien. CARGILL, dont le siège social est à Minnéapolis, et dont le P.D.G. à l'époque s'appelait Monsieur MacMillan, n'avait pas d'affection particulière pour les Bretons.

On a donc vu apparaître une pénétration très directe et très directive du capitalisme industriel dans l'agriculture, le plus souvent en tandem avec le capitalisme financier, surtout dans la deuxième phase, à partir des années 65 , lorsque UNICOPA s'est formée : les coopératives de Landernau, les petites coopératives des Côtes du Nord, du Finistère, la Rurale Morbihanaise, la CANA d'Ancenis, la SICA de Chalans, dans le domaine avicole, dont j'étais le remarquable président (elle a fait faillite, mais ne vous inquiétez pas), quand elles se sont mises à se lancer dans ce type de production - aviculture, puis rapidement porcs et ensuite jeunes bovins (veaux) -, elles l'ont fait en tandem avec le capitalisme financier, dont il se trouve qu'il finançait à la fois les entreprises industrielles et la production dans le domaine agricole.

Cela se faisait bien sûr, et cela se fait toujours, avec un maintien de la responsabilité personnelle. Vous savez qu'on a des exploitations à « responsabilité personnelle » dans ce pays, ce qui représente le mode de production le plus avancé, comme chacun sait... On laissait aux gars la responsabilité des emprunts pour la mise en place de l'appareil de production, c'est-à-dire les équipements, les bâtiments, et on leur laissait la responsabilité des emprunts pour la mise en place des poussins, des aliments, ou des porcelets, des médicaments, etc. Ce qui était une manière de leur faire prendre la 
responsabilité de la totalité des pertes en cas de pépin dans l'élevage. C'est comme cela qu'on supprime en fait le droit de grève chez les paysans, c'est évident. Si moi, actuellement, j'arrête défaire des poulets, je perds 18 millions $^{5}$. Dans mon métier, la grève est hors de prix. Ce n'est pas abordable, cela coûte trop cher, il faudrait être riche pour faire des grèves. Et il y a eu des réactions fortes de paysans qui entendaient rester paysans, tout en ayant le sentiment de s'être profondément modernisés. Je crois que c'est cela la véritable naissance de ce qui sera " Paysans travailleurs ».

1964, c'est l'époque des premières grandes manifestations, des bagarres, une faillite retentissante: LE MELINER (120 millions de perte - c'était énorme, 120 millions de perte chez les paysans -). On était habitués, nous, chez les paysans, à des rassemblements énormes - compte tenu aussi de l'importance de la population rurale. Et traditionnellement - c'est une très vieille tradition - on ne s'embarrassait pas pour sortir de la légalité quand il y avait des manifestations, même si les gens votaient à droite après. Je me rappelle, dans l'affaire LE MELINER, on avait fait une marche à quatre mille, sur quarante kilomètres entre Languidic et Vannes, à trois ou cinq à l'heure, les uns à pied, les autres en voiture, et le préfet avait commis l'imprudence de nous laisser entrer à vingt-cinq. Il s'est retrouvé coincé avec des bureaux qu'on lui poussait sur le ventre, on lui a apporté le téléphone, il a téléphoné au Premier Ministre de l'époque, Pompidou, pour obtenir que les 100 millions soient pris en charge par l'Etat - ce qu'on a obtenu -.

17 C'était le genre d'action qui se menait beaucoup plus dans l'Ouest que dans le Puy-deDôme par exemple, pour parler d'une région où il y avait un gars connu comme Michel Debatisse. ${ }^{6}$ Cela nous a amené à avoir des réflexes de dirigeants. On était habitués, parce que issus de la JAC, à se considérer comme les dirigeants naturels, compte tenu de notre compétence, du milieu à qui on devait enseigner ce qu'il fallait croire et pratiquer pour être sauvé - un peu comme les moines quand ils défrichaient la Gaule. Et on a commencé à demander une autre politique. Dans notre esprit, demander la mise en place de groupements de producteurs, c'était se donner la capacité de négocier collectivement avec les industriels. En fait, les groupements de producteurs ont eu une toute autre fonction, ils étaient tout à fait destinés à faire autre chose. On demandait aussi une certaine protection légale. Je crois que si le petit bout de la loi de $1964^{7}$ a été inscrit par le camarade Pisani, ministre du Général de Gaulle, c'est aussi parce qu'il y avait à l'époque d'importantes bagarres et manifestations dans l'Ouest... On essayait aussi de lutter contre les orientations politiques qui se dessinaient et on n'hésitait pas à intervenir directement, à Paris, à Bruxelles, sans y être invités et sans l'autorisation de la FNSEA $^{8}$. On avait perdu l'habitude de demander à nos pères spirituels de Paris ce qu'il fallait penser des actions qu'on menait.

C'est la période des grandes bagarres, 1966, 1967, les 20000 personnes à Redon, les 15000 à Quimper, on faisait des grandes kermesses... Quand je dis 20000 à Redon, non, on était 22000 , puisqu'il y avait 2000 flics! Et là ils étaient mal placés, on avait le balast pour nous et ils s'étaient mis sous le vent en lançant leurs grenades lacrymogènes, ce qui fait qu'elles leur revenaient dans la gueule... C'est pour cela que le combat a commencé vers six heures du soir et s'est terminé vers minuit. Il y avait vingt blessés de chaque côté, mais les leurs étaient plus graves que les nôtres. C'est une période de grande bagarre, on foutait le camp à Bruxelles, on invitait Mansholt à faire un grand débat public devant quatre mille personnes. 
19 J'ai beaucoup aimé cette période, puisque j'étais secrétaire général, cela permettait de faire des grands débats, j'aimais. J'étais secrétaire général de la Fédération Régionale de l'Ouest qui regroupait les neuf départements Pays de la Loire/Bretagne, dans le cadre des neuf FRSEA. ${ }^{9}$ On recevait de temps en temps des coups sur les doigts de la part de Cafarelli qui était le Président, et surtout de Debatisse qui était déjà secrétaire adjoint. Ils venaient pour nous apaiser, ils faisaient des communiqués pour nous condamner, surtout quand on arrêtait un ministre - Olivier Guichard - et qu'on l'emmenait dans une ferme de force. Grosse période de tension.

On produisait intellectuellement aussi, et c'est là qu'on fonctionnait comme des grands technocrates. Il n'y a pas pire technocrate, il faut être honnête, qu'un travailleur qui a accédé à des responsabilités, qui se croit chargé de diriger ceux qui l'entourent. On faisait des contre-plans - contre-plan Mansholt, contre-rapport Vedel ${ }^{10}$ -. La technique du contre-plan était très pratiquée par le PSU par exemple, et je ne cache pas que j'étais au PSU ${ }^{11}$. On faisait des documents absolument fulgurants qu'on allait expliquer à la campagne, commune par commune, canton par canton. Et les gens étaient tout à fait surpris de nous voir si intelligents, ils sortaient de la réunion en disant: " on ne se rappelle plus exactement ce qu'il a dit, mais il a rudement bien parlé ». Comme quand on entend le curé en chaire.

Dans cette période, un axe central, le désir de sortir du corporatisme et d'engager le dialogue avec les autres couches sociales. Cela a été la grande période des unités "ouvriers et paysans». Entendons-nous bien, on ne se trompait point dans le type d'unité, et la manière de la faire naître : il s'agissait de discuter avec l'appareil au niveau le plus élevé possible de la CGT, de la CFDT - voire de FO en Loire Atlantique où il y a une fédé FO assez anarchisante - et on faisait de grandes déclarations communes. Quant à aller discuter avec les ouvriers de l'agro-alimentaire qui se faisaient copieusement matraquer par l'appareil industriel - autant que les paysans, largement autant - et qui étaient souvent interdits de droits syndicaux, cela nous paraissait tout à fait impensable, ce n'était pas cela le problème. Par contre, la grande unité ouvriers/paysans, pour développer un complexe sidérurgique dans la Basse Loire, heureusement qu'on ne l'a pas fait... - il n'y a qu'à voir Fos, divers trucs de ce genre.

C'était là aussi les grands meetings, 30000 personnes sur le Champ de Mars à Nantes, le terre-plein de Penhouèt à Saint-Nazaire, puis cela gagnait peu à peu les autres régions. La Loire-Atlantique a été le moteur; on tenait le secrétariat général des neuf départements, ça résistait, il y avait des conservateurs que cela effrayait dans les autres départements.

Alors, dernier aspect de nos préoccupations du moment, puisque d'une part les grands méchants groupes multinationaux étaient en train de nous déposséder de notre travail, et que d'autre part la responsabilité individuelle de l'exploitation était foutaise - les choses se passant au niveau industriel et bancaire -, on a connu dans cette période un entrisme fantastique dans la coopération et les organismes mutualistes. On est parti à la conquête du pouvoir économique qui, dans notre esprit, devait aller jusqu'à la construction de supermarchés "meilleur marché », contrôlés par la profession agricole .

On arrive vers la fin des années soixante : 1967, 1968, 1969. On commence à s'apercevoir que l'entrisme ne fonctionnait pas très bien, qu'il y avait une logique de développement interne aux entreprises, fussent-elles coopératives. Par exemple, pour combler leur retard en matière d'investissement - parce qu'elles étaient apparues tard sur ce marché 
de grande distribution et de production massive industrielle -, elles avaient besoin de prélever un peu plus sur le revenu des paysans, au nom bien sûr de la solidarité, au nom d'un slogan que j'ai véhiculé : «il faut s'acheter un tracteur avant de s'acheter une télévision, parce que si on a un tracteur, il pourra nous payer une télévision, mais si on a une télévision, elle nous paiera pas un tracteur ». Et plus c'est simple, plus ça passe. Alors il fallait augmenter le capital social, faire des excédents, donner des acomptes extrêmement faibles, en expliquant aux gars qu'il y aurait peut être un petit rappel, s'il y avait des excédents. Et puis quand on arrivait à la fin de l'année, l'appareil de direction disait: «c'est pas possible». Et surtout la banque disait: "vous devez augmenter vos capacités d'autofinancement». Et donc on transformait cela en capital social, pris de manière entièrement autoritaire, aux producteurs bien sûr. Alors on a commencé à s'apercevoir que la conquête du pouvoir économique par les paysans, cela ne fonctionnait pas très bien.

Et puis 68 est arrivé là-dessus. C'est une étape extrêmement importante. Les analyses que l'on avait faites et nos pratiques avaient eu peu de répercussions, en ce qui concerne la FNSEA. Par contre, elles avaient été mieux accueillies par le CNJA ${ }^{12}$ - par les centres départementaux ou régionaux déjeunes agriculteurs, en Rhône-Alpes, dans une partie de la région Nord-Pas-de-Calais, et aussi un peu en Midi-Pyrénées, beaucoup moins en Languedoc-Roussillon, et puis dans le Jura, en Franche-Comté... Et alors c'est dans le cadre du CNJA, pour exprimer toutes ces équivoques, que s'est posé le problème de présenter des hommes qui étaient plus ou moins en rupture avec l'appareil de la FNSEA. Du type Bernard Thareau, ${ }^{13}$ qui avait fait $48 \%$ des voix, lors de sa candidature, mais qui avait la véritable majorité: on avait des voix en fonction des cotisations qu'on payait, or le nombre des cotisations de l'Aisne, cette année là, dépassait le nombre total des jeunes, agricoles et non agricoles, du département...! Il avait effectivement la majorité, Thareau, lorsqu'il a été battu par Simon, cela doit être en 1969, ou en 68.

Mai 68 est arrivé, alors cela a été un coup de tonnerre pour nous. Assez spontanément, dans une région comme l'Ouest, on a essayé de tirer vers une liaison avec le mouvement qui naissait. Disons à peu près tous les CDJA de l'Ouest, tous sans exception, ont tiré vers des liaisons, les plus fortes possibles, avec ouvriers, étudiants, enseignants,...y compris des manifestations communes, entre le 20 et le 25 . Il y a eu des meetings communs dans à peu près tous les départements de l'Ouest au moment où les paysans ont manifesté.

27 L'ordre de la FNSEA était « de manifester » parce qu'on ne pouvait pas laisser passer un mouvement comme cela sans dire qu'on avait, nous aussi, besoin de gagner notre croûte, mais de ne pas dépasser le stade des manifestations cantonales, en précisant qu'il ne fallait pas aller dans les villes importantes où il $\mathrm{y}$ avait des ouvriers, des étudiants, et des enseignants. Il ne disaient pas qu'il s'agissait de crapules, mais il ne fallait quand même pas «se mélanger avec ces gens-là ». C'était l'époque où Debatisse allait voir le Général de Gaulle pour lui témoigner son affection.

Le point culminant de ce type d'action commune, concrètement, cela a été Nantes et l'envahissement de la ville. On est entré par quatre côtés, avec un tracteur en tête sur chaque grande route. On était 3 à 4000 à peu près, 1000 sur chaque point, avec des pancartes « Place au peuple "qu'on a portées jusqu'à la Place-Royale qu'on a débaptisée et qu'on a appelée «Place du peuple». La Place-Royale c'est la plus belle de Nantes, bien sûr. On a fait une jonction avec les ouvriers et les étudiants. Et les jeunes paysans 
ne sont pas innocents - ils n'étaient pas les seuls - du fait que la Préfecture brûlait le soir. C'était assez spectaculaire de voir la partie toute neuve de la Préfecture en feu...

Donc il y a eu un certain mouvement mais c'est surtout les retombées qui sont importantes. Jusque là, on n'avait pas changé d'attitudes ni de méthodes, ni de manière d'agir, ni d'analyse. On avait seulement été très sensibles, on avait été présents. Ce qui nous a secoués, quelques mois après, c'est une série d'articles parus dans la revue "Frères du Monde », de Bordeaux ${ }^{14}$ - qui étaient inspirés par des jeunes issus du MRJC. ${ }^{15}$ Alors qu'on avait été aussi les acteurs de mai 68, ils remettaient en cause nos pratiques antérieures, notamment nos pratiques bureaucratiques. On voyait ça d'un sale œil! C'étaient des gars comme Bertin, Jean Bernard Mabilée et d'autres. Ils remettaient en cause nos pratiques bureaucratiques, technocratiques, manipulatoires à l'égard des gens, qui nous faisaient - disaient-ils ajuste titre - complètement négliger le fait qu'il y avait dans nos communes des paysans qui se cassaient la gueule tous les jours, qui étaient victimes de cumuls... - on n'avait pas le temps, on avait tellement de trucs à faire avec Mansholt... - ; qui nous faisaient négliger aussi le type qui était mis en faillite par une entreprise, surtout si elle était coopérative. On n'allait tout de même pas critiquer la coopération, alors qu'on faisait de l'entrisme, on n'allait pas reprocher à des paysans de s'être laissés intégrer par des capitalistes... comme si on allait reprocher à un travailleur de travailler dans une usine qui ne serait pas une coopérative ouvrière... C'est complètement fou, bon, on était comme cela. Cela faisait mal, très mal, moi personnellement cela m'a profondément heurté, je trouvais cela très injuste... parce que très vrai. Qu'il y ait eu des outrances dans leur expression, c'est peut-être vrai aussi, mais ils nous ont très très secoués.

C'est à cette époque-là que s'est déclenché un événement assez considérable. Cela doit être en 1970, la première grande action qui nous a conduit concrètement sur le terrain à remettre en cause la coopération : la grève du lait. La grève du lait mérite à elle seule une étude particulière, elle a vraiment beaucoup marqué parce que pour la première fois des paysans se battent sur le slogan : « le prix du lait doit payer le travail ». Je crois qu'on avait calculé, sur la base de 500 litres par vache et par an : quand on déduit toutes les charges, voilà ce qu'il faut avoir pour que notre travail soit payé, et voilà le prix du litre de lait permettant de payer ce travail. Et les quatre derniers centimes, vus sous cet angle-là, sont tout à fait essentiels, c'est évident. Je crois que l'on demandait 7 centimes et que la négociation s'est terminée à 4 . Et pour l'obtenir... vous allez voir à quel prix on se lance dans la grève... Heureusement en Bretagne il y avait encore pas mal de barattes et d'écrémeuses, une partie du lait a pu être sauvée. En Pays de Loire c'était déjà beaucoup moins vrai, l'industrialisation étant plus ancienne, il $\mathrm{y}$ a eu près des trois quarts du lait perdu. C'est une période, cette grève, où tu travailles forcément beaucoup - faut traire les vaches et puis faut faire les manifestations dans la journée et où tout ce que tu sors, tu le perds. Tu ne perds pas que ta journée : la production d'une journée de lait, ça sert aux amortissements, aux engrais, au paiement du matériel...la part qui reste pour payer le travail est très faible. On avait même compté à l'époque qu'une journée de grève du lait correspondait à sept jours de paiement du travail. La grève a duré 13 jours, c'est-à-dire qu'il y a eu 100 jours de grève. C'est tout à fait énorme, 100 jours de perte du revenu net.

31 Et la grève s'est appliquée aux coopératives. Là il y a eu un tri fantastique. Petite anecdote pour illustrer cela : il y a eu chez moi, un soir, une réunion de 35 personnes avec Bernard Thareau qui était un bon copain. Il a fait son choix ce jour-là . Les grèves 
totales avaient lieu dans le Finistère, le Morbihan, les Côtes-du-Nord et quelques zones ailleurs. Mais pas de grève totale à Ancenis. ${ }^{16}$ On lui a dit: " On ne te demande pas la démission totale du Conseil d'Administration de la coopérative d'Ancenis, puisque tu n'as pas l'air de le vouloir, mais demain on sera 4000 à manifester » (il s'est fait d'ailleurs enfermer le lendemain, il n'y a pas eu de détails là-dessus). «Et on te demande au moins de déclarer que tu abandonnes toutes tes fonctions et que tu passes du côté des tiens, tant que le minimum de 4 centimes ne sera pas donné ». Il a dit non. « Non, parce que en participant de l'intérieur, je peux rendre service individuellement à des gens". Il le disait de très bonne foi, Bernard est un type humainement extraordinaire, très intéressant, très attachant. « Non, je reste pour la gestion ».

C'est la première fois qu'on a publiquement fait dresser des paysans contre leurs coopératives. Je crois que c'est une rupture tout à fait fondamentale et qui demeure, qui aujourd'hui encore demeure. Voilà, si vous voulez une première version des choses, c'est un peu décousu ce que je vous dit, mais enfin on est trop cartésien en France, il faut s'habituer à cela - Dans le même temps, chez nous, les débats continuaient. Moi, c'est à ce moment-là, ayant fait une première crise cardiaque - maintenant cela ne m'effraie plus puisque j'ai l'habitude - je pensais qu'il fallait que je fasse un cadeau à mes adversaires, les Debatisse et les autres, c'est à eux que je pensais surtout. Je me suis dit, il faut que je fasse un bouquin. Je l'ai commencé dans l'hiver 68-69, et j'étais tout à fait convaincu qu'il pourrait sortir au printemps. Bon, il m'a fallu pas loin de deux ans pour le faire, «Les paysans dans la lutte des classes $~^{17}$ est sorti.

La première rupture au sein du CNJA s'est faite à ce moment là avec l'« Interdépartementale ", regroupant un peu plus du tiers, 35 à $40 \%$ des départements. Le plus gros de la troupe des militants des «Paysans travailleurs » est issue du CNJA. Dans la Fédération régionale de l'Ouest quelques-uns ont suivi, mais très peu. Ils ont été tout à fait effrayés par le pas que cela représentait, qui consistait simplement à s'organiser sans avoir encore réellement quitté la structure, mais qui était le premier pas vers le fait de quitter la structure syndicale.

Alors dans les groupes qui pensaient à cela, notamment ceux issus du MRJC, doublés d'intellectuels de l'INRA - pour ne pas les nommer, Gervais, Servolin et Coulomb à l'époque, Nallet n'y était pas -, dans ces groupes là, on s'est retrouvés, on a pensé qu'il fallait sortir un certain nombre de documents écrits, voire une petite presse. Les CDJA dissidents avaient un journal qui avait été fondé à l'Ouest - « Vent d'Ouest » qui existait depuis 1966 ou 67. Et d'autre part, il y a une feuille qui est parue «Paysans en lutte", qui a dû naître en 69. J'étais parmi ceux qui la rédigeaient.

Des conflits importants dans « Paysans en lutte » sont apparus dès le départ. Il y avait en gros deux orientations qui se dessinaient. La première, la plus à gauche - tout au moins elle se décrivait comme telle -consistait à dire : Ce qu'il faut, c'est créer les bases d'un vaste mouvement qui s'intégrera et apportera des travailleurs aux forces politiques qui ne vont pas manquer d'apparaître après tout le bouillonnement de 1968; il faut un mouvement et les travailleurs n'ont pas besoin d'une multitude d'organisations, ils n'ont besoin que d'une organisation politique qui leur permette de réfléchir et d'agir sur tous les problèmes qui leur importent, et c'est bien sûr eux qui doivent en avoir la direction. Il ne faut pas créer d'organisations syndicales qui seraient forcément corporatistes, il vaut mieux agir dans les structures syndicales existantes.

Réfléchir et agir sur tous les problèmes, y compris les relations homme-femme, l'existence ou non d'un couple, le baptême ou non des enfants, etc., dans l'Ouest cela a 
fait quelques petites étincelles. Si bien qu'on se réunissait entre paysans exploités, ayant des problèmes, face à l'exploitation économique dont on était l'objet, paysans remodelés par le monde du capital pénétrant de plus en plus intimement l'acte de travail. Et on n'avait pas le temps de discuter de cela, parce que les autres nous entraînaient dans tel ou tel débat, avec tous les arrières fonds culturels qu'il y avait. Et l'on n'avait pas avancé d'un seul millimètre, si ce n'est qu'il y avait un peu moins de militants à chaque fois, parce que les gars en avaient plein le... dos. Ils n'y revenaient point, et cela ne leur paraissait pas régler les problèmes quotidiens auxquels ils étaient affrontés.

C'est l'époque aussi des longues marches, les «Paysans travailleurs » qui naissaient faisaient l'objet de longues marches où des gens venaient nous dire ce qu'on était, ce qu'il fallait qu'on devienne, et cela s'est traduit par un slogan qui a été inscrit à la tête d'un article de « Paysans en lutte »: « Les syndicats attirent tous les éléments pourris et pourrissent tous les éléments sains ».

Du point de vue doctrinal, c'est un article de Marcel Colin, il y a aussi un article extrêmement curieux, qui doit être dans le numéro 3 ou 4, expliquant qu'il fallait que les paysans soient les supports des futurs maquis. C'est une période spéciale aussi, il faut bien le dire, l'après-mai.

Et puis il y avait une autre ligne dans laquelle je m'inscrivais bien plus, personnellement, qui consistait à dire: On n'échappera pas à la création d'une organisation syndicale, les méfaits de la bureaucratisation sont réels, mais il faudra être clair à l'égard des paysans, trancher, on ne peut pas continuer ce que l'on a fait, tenir des appareils, mobiliser des foules impressionnantes qui nous entendent et qui s'en foutent, qui mènent des actions qui peuvent être intéressantes, mais qui n'avancent pas d'un millimètre par rapport à tous leurs schémas classiques. Si on pense que les paysans, par des éléments de leur vie de travailleurs - malgré toutes les équivoques - peuvent être des agents actifs de la lutte des classes, il faut s'organiser pour être présents dans la lutte des classes, cesser la comédie de la présence coopérative et de la conquête du pouvoir économique, cesser la comédie de l'interprofession des groupements de producteurs, discutant comme des partenaires égaux avec l'industriel, mieux encore jouant le rôle d'écran entre les producteurs et les industriels. Parce que c'est leur véritable rôle, de structure écran qui doit régler les conflits, mais qui n'a pas d'argent pour le faire. Mais juridiquement on va même jusqu'à leur donner le pouvoir de payer les producteurs, ce qui protège singulièrement celui qui nous exploite, en réalité. Tout cela il faut le remettre en cause, mettre en place une organisation syndicale. Bien sûr, il n'est plus question d'avoir des permanents à vie, d'avoir notre Maurice à nous, qui est mineur encore trente ans après - parce que c'est pas vrai qu'on est encore mineur trente ans après, pas plus que paysan d'ailleurs -. Il y aura des collectifs à tous les stades, sans hiérarchie, renouvelables à tout moment, et au maximum pour une période de deux ou trois ans - c'est comme cela que fonctionne « Paysans travailleurs »-. Alors on a fait beaucoup d'anti-intellectualisme à l'époque, et puis les vieux crocodiles devaient se mettre sur la touche, j'étais un peu visé, cela ne fait pas de mal d'ailleurs défaire des petites cures comme cela. Mais notre option pour un syndicat en rupture avec les institutions agricoles était inscrite.

Elle s'est exprimée pour la première fois de manière collective dans ce pays lors de la première marche sur le Larzac qui a été prise en charge publiquement, officiellement, par " Paysans travailleurs ", aidés par d'autres mouvements, en réaction 
contre la FNSEA qui avait demandé d'arrêter la marche des paysans sur Paris à Orléans, pour qu'ils ne viennent pas foutre la merde à Paris. Et pour qu'ils ne viennent pas non plus au contact de la "pourriture urbaine ", c'est-à-dire des contestataires, des révolutionnaires... C'est une mécanique qui est toujours la même. L'équipe de «Paysans en lutte ", qui était déjà relativement atomisée et peu influente sur le terrain, a été très hostile à l'initiative qui consistait à organiser une marche des paysans sur le Larzac, ou au moins à ce que " Paysans travailleurs » en prenne la direction, parce que c'était le rôle d'une organisation politique et non pas d'un mouvement comme le nôtre, qui n'était qu'une pierre d'attente d'une autre affaire qui viendrait bientôt.

41 Cette hostilité n'a rien d'étonnant puisque la rupture avec la première tendance de «Paysans en lutte » avait eu lieu, notamment quand on a choisi le titre de la nouvelle organisation, parce que déjà cela devenait une organisation syndicale. "Paysans travailleurs ", c'était déjà un symbole. On n'était pas les premiers à l'utiliser: il n'y avait pas de $\mathrm{MODEF}^{18}$ avant la guerre, il y avait « Paysans travailleurs ", lié au P.C. Après ce choix, il y a eu encore quelques numéros, puis «Paysans en lutte», par manque de lecteurs, a fermé les portes.

"Paysans en lutte " était très hostile à cette marche sur le Larzac où l'on voulait s'affirmer en tant que groupe social, mais de manière non corporatiste, rejoindre des problèmes posés non seulement aux paysans mais à l'ensemble du pays, le problème de l'utilisation de l'espace, de la liaison ouvrière, de la liaison Lip/Larzac, de la production d'armement, de la destination du sol, d'une manière générale. On est apparu là la première fois, c'était en 1973, je crois. On s'est un peu structuré. Après «Paysans travailleurs » a connu toutes les crises qu'ont connu les groupuscules en France: les tentatives de main mise, qu'elles viennent de la Gauche Prolétarienne, du PSU, de l'Humanité Rouge,... ont été fort nombreuses.

Où se situe «Paysans travailleurs $»^{19}$ aujourd'hui ? Hommes, femmes, 6 à 7000 militants, une influence qui peut mener à des actions autour de 60 à 80000 personnes dans des périodes de tension sur de grands événements, du type de la grève du lait, du type Larzac... Une influence qu'on peut situer autour de 6 à7 \% des paysans. C'est la plus jeune des organisations, elle ne vient que maintenant d'adopter le principe des statuts syndicaux, il a fallu du temps pour que cela se matérialise, même si les décisions étaient déjà prises en pratique. C'est la plus petite des organisations. Au niveau des plus jeunes, je crois que son influence est plus élevée. La moyenne d'âge des paysans, c'est quand même 54 ans.

FB. : Qu'est devenu le courant contestataire interne aux FDSEA?

B.L. : Les FDSEA dissidents n'ont abandonné ni le Crédit Agricole, ni la coopération, ni la perspective d'une conquête du pouvoir économique. Mais ils ont mené des actions qui sont très chouettes. Curieusement, comme les durs de "Paysans en lutte ", ils se sont tous retrouvés au PS... Faut ce qu'il faut pour faire la Révolution. La Fédération de Loire-Atlantique a été tellement loin contre la FNSEA et ses complicités avec le pouvoir qu'elle s'est fait jeter dehors, elle a été expulsée. Ce qui fait qu'énormément de paysans qui auraient été à "Paysans travailleurs ", s'il n'y avait pas eu ce type de fédération, sont restés à la Fédération dissidente. Ce qui fait qu'en Loire Atlantique, on est 3 à 400 militants, alors qu'on pourrait en avoir au moins un millier. Et, curieusement, en Vendée, «Paysans travailleurs » est relativement plus fort parce qu'ils ont la chance d'avoir Auguste Grit, secrétaire général de la FNSEA, qui est un homme de droite très affirmé, très clair. Dans les Côtes du Nord, c'est un peu le même phénomène et il n'y a 
que 6 à 700 militants. Par contre, dans le Finistère, où il y a un potentiel énorme, JeanNoël Leduc fait écran et, à l'inverse, dans le Maine et Loire où la fédération est très à droite, « Paysans travailleurs » se développe beaucoup plus.

(Dans un deuxième temps du séminaire, Bernard LAMBERT approfondit certains points portant soit sur l'organisation de " Paysans travailleurs ", soit sur leurs actions).

F.B.: Quel bilan fais-tu des luttes aux côtés des ouvriers, depuis le moment où, vers 1965 , votre souci était de rejoindre leurs états-majors ? Est-ce qu'après 68, il y a eu de votre part d'autres formes de rapprochements avec les ouvriers?

B.L. : Oh ! Que oui ! Cela a été un axe central, et cela a carrément commencé en mai 68 , où il $\mathrm{y}$ a eu des renversements extrêmement douloureux en provenance des organisations syndicales ouvrières des entreprises agro-alimentaires, quand il s'est agi de prendre plusieurs décisions comme alimenter les élevages, parce que les garces de poules, quand elles ne sont pas alimentées pendant 48 heures, elles crèvent, c'est pire qu'un haut-fourneau. Est-ce que les dockers allaient décharger les tourteaux de soja? Est-ce que les usines d'aliments du bétail allaient fonctionner? Qu'est-ce qu'on allait faire de la production? Alors il y a eu des engueulades terribles, et des empoignades effroyables, d'ailleurs contre la volonté de Gilbert Declerc (la CFDT était majoritaire dans Vagro-alimentaire) et d'autres mecs avec qui on marchait à fond la main dans la main. On contrôlait la ville, on distribuait l'essence, on en refusait d'ailleurs au secrétaire de la Préfecture qui était venu nous en demander, il avait eu tort... Mais les mecs de Vagro-alimentaire disaient: «Maintenant, on va causer, parce que vous êtes nos dirigeants, vous êtes dans les Conseils d'administration, à quoi on joue ? »Cela a été dur et, globalement parlant, compte tenu de la pression des appareils ouvriers, voilà le résultat: on a fait tourner les élevages, on a distribué les carburants pour le ramassage des foins, etc. mais les productions ont été distribuées aux ouvriers jusqu'à Billancourt (c'est les gars de l'agro qui alimentaient Billancourt, cela leur a bien passé depuis). Le principe étant qu'on apportait une masse de produits à prix coûtant, et qu'on s'arrangeait pour qu'il y ait un volant à distribuer gratuitement. Les écoles servaient de supermarché autour de Nantes.

49 Cela a été le premier pas du redressement et puis après on a vraiment cherché, notamment dans l'Ouest, le contact avec les ouvriers à chaque fois que cela a été possible. Alors les résultats ont été très inégaux. Cela s'est fait des deux côtés. Pour annoncer une grève des ouvriers de la coopérative agricole d'Ancenis, chaque chauffeur de camion de lait a été accompagné d'un paysan durant toute sa tournée pour distribuer des tracts. Des tracts où on fusionnait les revendications des ouvriers et des paysans et où on présentait les véritables problèmes, ce qui fait que la direction a cédé avant la grève. A elle seule, l'arme de l'unité avait joué pleinement. Au contraire, je me souviens d'une autre firme laitière, Eric CAMBON - le groupe ATLA-LA1T - où le refus de liaison de la $\mathrm{FGA}^{20}$ avec les "Paysans travailleurs", pour des raisons doctrinales, a conduit à une grève suicide avec échec total à la clé. Les cadres ont pris les camions, quelques paysans de droite aussi, les autres laiteries ont été toutes solidaires pour écouler la marchandise et la grève est tombée. On a voulu rattraper, après c'était fini.

50 Mais je me souviens surtout d'une bagarre avec la laiterie ENTREMONT de Quimper: après avoir berné les paysans en se servant des ouvriers qui venaient d'obtenir une augmentation de $35 \%$ de salaire (Accords de Grenelle, ils étaient au SMIG avant, ils y étaient encore après), en disant: «je ne peux pas payer plus cher les paysans », puis 
après avoir berné les ouvriers en leur disant « le titrage au km est quatre fois inférieur à celui de la Hollande, le lait c'est sale, on ne sait pas qu'en foutre, on ne peut pas faire de fromage, etc. » au dernier moment, et de manière tout à fait inattendue, une grève a démarré alors qu'il n'y avait pas de syndicat. Trois cents ouvriers. Alors que le patron avait lancé des appels aux paysans, et que certains arrivaient avec leurs permis poids lourds pour conduire les camions. Un autre groupe de paysans a dialogué tout de suite avec les ouvriers, fait un tract commun; puis ils sont partis sur les camions avec les chauffeurs pour distribuer les tracts, en décidant que les cuves seraient remplies mais que le lait ne serait pas traité, ce qui fait que le patron était obligé de payer. Quand les cuves seraient pleines, on en mettrait directement dans l'Odet qui passe à Quimper. Il avait blanchi, l'Odet! En 24 heures, le patron a plié sur les revendications qui s'étaient unifiées au dernier moment...

51 Il y a eu des tentatives, avec des réponses très inégales de part et d'autre, d'autres champs d'action commune qui se sont ouverts récemment, comme, avec les consommateurs, le problème du veau.

C'est une période de l'histoire pour laquelle je ne suis peut être pas très objectif, je vous la dis de manière non ordonnée, je l'ai vécue très intensément. Cela foisonnait sec... alors je vous la rends comme je l'ai vécue.

53 N.M.: Quelles sont les principales différences entre "Paysans travailleurs » et le MODEF ?

54 B.L. : Les différences entre les «Paysans travailleurs" et le MODEF, c'est assez considérable. Il suffit de voir leur presse, et les ouvrages théoriques auxquels ils font volontiers référence, comme les travaux de Perceval : le MODEF n'a jamais cru que les paysans pouvaient être eux-mêmes, de par leur condition de travailleurs, des agents de la lutte des classes.

Le thème, c'est qu'ils sont paupérisés, appauvris, évincés de leur métier, qu'il faut en conséquence venir à leur secours en faisant des prêts à $1 \%$ sur trente ans pour acquérir la terre. Quand je vois cela, moi cela méfait très mal, alors que la propriété du sol, c'est bien l'aliénation majeure du milieu paysan... Et économiquement parlant, c'est par là que passe le transfert des capitaux qui sortent du secteur primaire au profit des secteurs secondaire et tertiaire, notamment au profit d'une urbanisation des banlieues venant des héritages, etc. La terre, la propriété du sol, je crois qu'il faut la remettre en cause totalement. Le MODEF continue à se comporter comme si les paysans étaient à un stade d'économie précapitaliste, ils s'adressent à eux pour se faire des alliés, pour que "Jacquou le Croquant» ne devienne pas fasciste. Parce que c'est une éventualité non négligeable. Les chemises vertes de 1936, dans ma région, je connais - pas moi directement, je n'avais quand même que cinq ans - mais cela a existé.

A partir du moment où le travail a été divisé, où la production s'est spécialisée, où l'acte de travail a été contrôlé, y compris par contrat, chez le paysan lui-même - contrôlé par le contremaître qui s'appelle technicien agricole -, à partir du moment où les choix de production sont entièrement entre les mains de la banque, et non pas entre les mains du paysan qui s'installe, puisque l'accès aux prêts en dépend, et c'est eux, non pas le paysan, qui font les budgets provisionnels... - et cette banque est en liaison avec l'agroalimentaire qui pose un certain nombre de conditions et de destinations aux produits -. Donc à partir du moment où l'on est en face d'un homme qui n'est pas reconnu en tant que travailleur (il n'a qu'à perdre son métier, il n'a pas droit au chômage), mais qui n'est pas non plus reconnu par les capitalistes comme entrepreneur, puisqu'il n'a 
même pas le droit de déposer son bilan (il est responsable indéfiniment, y compris sur ses biens personnels, ceux du ménage, de tout ce qui peut arriver sur l'exploitation)... à partir du moment où l'on est devenu un type qui n'établit jamais de factures, même s'il en reçoit tout le temps (c'est quand même un drôle d'entrepreneur, même le petit artisan de chez moi il méfait une facture ..) on est devant une modification substantielle.

Je ne dis pas qu'on est des salariés, mais il y a une modification substantielle du statut du producteur, quoiqu'en dise Servolin, mon ancien (inaud.)... de gâche. Pour être clair, je pense qu'une analyse marxiste doit se renouveler puisque la condition de cette partie du monde du travail s'est modifiée. Et je crois qu'il y a des éléments, dans cette condition de travailleur, qui sont porteurs de lutte des classes, ce qui est extrêmement nouveau, c'est là-dessus qu'on diffère profondément avec le MODEF. Et quand je suis méchant, je leur dis: "vous leur promettez qu'ils pourront devenir des koulacs, et qu'est-ce que vous en ferez demain?»

Qu'il y ait des contradictions, qu'on baise le paysan en le faisant devenir propriétaire et par conséquent, dans sa tête, petit spéculateur, d'accord...Mais on peut faire comprendre maintenant à de plus en plus de paysans que devenir propriétaires est totalement et éternellement illusoire. J'achète le tracteur, j'ai le prêt à cinq ou sept ans, au bout de cinq ou sept ans - et c'est calculé pour - je dois racheter un autre tracteur, je n'en suis jamais propriétaire. J'achète un poulailler, j'ai mon prêt de douze ans, au bout de douze ans, soit il est frappé d'obsolescence, dépassé techniquement, soit les coûts d'entretien sont équivalents aux charges que j'avais pour le remboursement de mes emprunts. J'en sais quelque chose. Je me suis installé en 1951 et je suis toujours propriétaire de mes dettes, à ne pas savoir qu'en foutre. La propriété, par contre, d'un troupeau laitier, qu'on oblige actuellement à accumuler, au prix de serrage de ceinture, c'est une propriété réelle, avec une valeur qui va en augmentant. Je ne crois pas qu'elle le demeurera éternellement. On était propriétaires de nos poules, on ne l'est plus. On était propriétaire de nos truies, des porcelets, on ne l'est plus ; on était propriétaire des veaux, on ne l'est plus, on était propriétaire des jeunes bovins, on ne l'est plus. Il y a des sociétés, y compris des coopératives, qui mettent des brebis en location. Et le Crédit Agricole de Loire-Atlantique doit être rendu à 1000 warrants renouvelables automatiquement sur les troupeaux de vaches. Il n'y a plus, dans ce cas, de propriété de vaches ni de génisses, puisque le Crédit Agricole a la garantie de se payer sur les vaches qu'il possède par warrantage, au regard de la loi. C'est une évolution qui n'est pas prêt de s'arrêter.

En ce qui concerne le sol, je vous parle toujours des petits paysans, je ne vous parle pas de Philippe Neeser, ${ }^{21}$ c'est un autre secteur que l'on traitera une autrefois. Les petits paysans sont contraints à deux achats importants pour les engager dans la voie de la productivité. Le plus difficile et le plus lourd de ces achats, c'est l'achat de la maison. Même quand on prend une terre en fermage, on sépare maintenant les bâtiments des terres. Cela devient de plus en plus courant. Il faut acheter sa maison, il n'y a pas de locatif à la campagne, on n'y échappe pas. Il faut aller voir la banque pour construire je ne parle pas des paysans qui sont venus au monde tout habillés -, il faut aller voir la banque, il faut donc faire une production. C'est pour cela que les dix chèvres, les trois biques dans les montagnes de l'Ariège, mon petit œil et ma grosse dent, si cela peut marcher, je ne suis pas contre des expériences, mais globalement parlant, c'est impraticable. Que des témoignages existent, je suis pour, mais il ne faut pas déconner, 
là on investit dans la maison, il faut donc faire une production, s'engager dans la voie du productivisme, du travail dingue. Et la banque met des conditions.

C'est un moyen de rendre les mecs productifs et de leur faire accepter de ne pas être rémunérés de leur travail. La femme ou le mari ira travailler dehors pour constituer le revenu. Moi, ma femme travaille au noir chez des maraîchers, à arracher des légumes pour qu'on puisse gagner notre croûte. J'ai l'équivalent de 12 hectares avec mon poulailler et on est heureux d'avoir de quoi rembourser nos emprunts, c'est une grande joie. Oui, on nous motive au-delà du paiement du boulot, c'est prodigieux.

Alors à cause du sol et de la maison, tout le monde dit «j'ai quelque chose à protéger Vive Giscard - parce que quand même, je suis propriétaire». Politiquement, cela fonctionne de manière impeccable et quand le paysan disparaît, le fils qui reste est aux prises avec les parts à verser à ceux qui sont partis. Une enquête partielle montre que l'habitat suburbain est financé à $52 \%$ par l'argent des successions. Il s'agit d'un véritable transfert en faveur de plusieurs secteurs économiques, et d'abord des spéculateurs sur les terrains à bâtir et la construction.

Inutile de vous dire qu'entre frères et soeurs, c'est la charité chrétienne avant, après c'est la joie, l'héritage, sacré salaud... tu vois ben... Et le gars en prend pour toute sa vie pour devenir, finalement, lui aussi, propriétaire. Et si tout le monde fout le camp, celui qui rachète, parce qu'il y a l'impératif de s'agrandir (la surface minimum d'installation augmente...) il fait la même chose, c'est le canal par lequel on fait de l'accumulation de capital au détriment du secteur primaire, un des canaux essentiels. Ça, et puis l'élevage des gamins...

63 Cette analyse m'amène à dire que tout est profondément illusoire. On dit au gars : «tu vivras très pauvrement, mais tu mourras riche", avec toutes chances d'ailleurs qu'il meure pauvre. Alors c'est là que je me sépare profondément des analyses du MODEF.

Sur certains points et certaines luttes concrètes, les conditions sont parfois remplies pour mener des alliances, parfois même très larges : par exemple, dans les luttes sur la taxe de coresponsabilité dans le domaine laitier, ${ }^{22}$ chacun donnant les explications dans la zone d'influence qu'il a. On peut même être parfois unifié avec le MODEF - qui ne va pas toujours à la recherche d'alliance et d'unification, c'est plus souvent le contraire, car il joue souvent le splendide isolement.

65 F .B. : De plus, géographiquement, il doit être implanté dans des zones où vous n'êtes pas?

66 B.L. : Exactement, il est à la fois dans des zones d'agriculture plus traditionnelle et de tradition jacobine.

67 N.M. : Mais on avait pourtant dit que dans l'Ouest il y avait eu une progression du MODEF?

68 B.L. : Une progression aux Chambres d'Agriculture, mais dans la structure syndicale, absolument pas. Par rapport à nous, ils sont groupusculaires dans l'Ouest. Par ailleurs, des alliances sont possibles aussi avec le MSTT $^{23}$ et avec plusieurs petits groupes: Savoie, les fermiers de la Manche, fermiers et métayers séparés de la FDSEA et de la FNSEA. La Savoie se trouve sur les mêmes bases que «Paysans travailleurs ", mais ils n'ont pas osé se proclamer " Paysans travailleurs ", par crainte qu'on agite le drapeau rouge devant les populations. De son côté, le MSTT représente une scission qui s'est faite dans "Paysans travailleurs ", à un moment où, principalement, le PS souhaitait que « Paysans travailleurs » devienne sage, rangé, et rentre dans le rang. Gaborit là- 
dessus était très clair. Groupent-ils 500 paysans ? Des alliances sont donc possibles entre nous, les petits groupes et une partie du MSTT. On a aussi des alliés potentiels avec les fédérations de gauche de la FNSEA. pensé à nous, pour nous faciliter les choses, à travers une loi "Sécurité/Liberté ». Ce sera liberté chérie pour nous... Pour occuper un Conseil d'Administration de coopérative sur des problèmes concrets et le plus souvent collectifs, on n'a aucune alliance à attendre, ni des Fédérations de Gauche, ni du MODEF. On fait d'autres alliances à l'extérieur, on a fait alliance avec des organisations de consommateurs. Je précise que cela n'a pas été l'UFC, ${ }^{24}$ alors qu'on s'était vus avant le déclenchement de la bagarre veau. Cela a été essentiellement avec la $\operatorname{CSF}^{25}$ et la $\operatorname{CSCV} .{ }^{26}$ On en reparlera à propos du productivisme, c'est un secteur où il va falloir travailler très très fort.

72 F.B. : Donc tu fais une sorte de hiérarchie dans les types d'enjeux de luttes au niveau des alliances?

73 B.L. : Absolument. De même que quand on remet en cause la propriété, on n'a pas le MODEF avec nous. Si on veut charruer des terres ${ }^{27}$ parce qu'il y a eu une distribution injuste, ce n'est pas une pratique courante, on ne l'a jamais fait en commun. Les FDSEA de gauche viennent, mais toujours très timidement.

Avec la loi "Sécurité/Liberté », on aura droit à cinq ans de cabane si l'on occupe des locaux d'entreprise qui ne sont pas notre propriété - bien que ces coopératives, et le Crédit Agricole, soient pratiquement à nous, elles ne sont pas juridiquement à nous. Nous, nous n'avons pas l'arme de la grève, on ne renouvellera jamais la grève du lait, à cause du volume de production et parce qu'il n'y a plus d'écrémeuse. Il y a sept ou huit ans, alors que trois ou quatre paysans sautaient, on a pris en otage Monsieur X., le Directeur de la coopérative de Landerneau, qui gagnait comme vous et moi autour de 5 millions par mois. Cela s'est terminé dans la forêt, il a fait huit kilomètres à pied (il y avait longtemps, d'ailleurs, que cela ne lui était pas arrivé). On n'a vraiment fait aucune 
violence, mais, avec la nouvelle loi, alors là, ça coincerait. Ça pourrait bien être de l'ordre des Assises. On a pas mal bossé là-dessus et on voit avec terreur les applications.

75 Sébastien coX.: Votre analyse est juste sur la place dépendante des paysans producteurs dans le cadre de l'intégration vis-à-vis de l'industrie, des coopératives et des sources financières. Cela m'étonne que dans votre perspective d'alliances, vous ne considériez pas ceux qui sont vos alliés naturels à l'intérieur de ce pouvoir, c'est-à-dire les travailleurs de Vagro-alimentaire, les salariés des coopératives, les travailleurs des banques, des services, les techniciens,...

76 B.L. : Si. Je n'ai pas voulu m'y étendre, parce que je l'avais pris comme exemple, antérieurement, dans l'affaire du lait. Je pourrais te donner l'exemple de la grève des employés du Crédit Agricole de Vendée. Si elle a été gagnée et si elle a pu se maintenir, alors qu'Auguste Grit mobilisait toutes ses troupes pour réoccuper les locaux, faisant appel notamment à MANPOWER pour les intérimaires dans le domaine de l'informatique, ce sont les «Paysans travailleurs » qui ont permis que cela soit gagné. Les salariés du Crédit Agricole n'ont pas eu à supporter le poids de la manifestation anti-grève organisée par Grit, puisque c'est nous qui avons fait le premier cordon.

F.B. : Y a-t-il eu dans cette période des divergences au sein de " Paysans travailleurs »?

B.L. : Pour faire court, j'en vois de deux sortes: «dans le monde paysan, il y a les petits, les moyens, les gros ${ }^{28}$ - comme dans les pommes de terres, il y a les moyennes et les grosses -, "les petits sont condamnés à disparaitre, les moyens, la moitié deviendront petits et disparaîtront, et l'autre moitié rejoindront les gros et deviendront capitalistes ", c'est la seule explication qu'on donnait à la présence du capitalisme en agriculture, elle nous paraît courte. C'est une explication qui fonctionnait déjà à un autre moment de l'histoire - en 1917 c'était sans doute la seule explication - mais aujourd'hui il y a d'autres modes de présence du capitalisme en agriculture et je n'accepte pas ce jugement moral.

J'ai un copain, près de chez moi, trois hectares de terres, il veut s'installer, lui, sa femme, jeune « Paysan travailleur ». Il discute avec le Crédit Agricole d'Anjou, envisage divers types de production. On ne lui laisse d'ailleurs pas le choix. Il fera de la multiplication, de la sélection pour les poulets - cela n'existe pas seulement pour le maiss. Pour les animaux, il n'y a que sept sélectionneurs dans le monde, tous tenus par l'industrie pharmaceutique - INRA, Institut Mérieux, plus subventions. Il veut faire construire une maison, sur ces trois hectares, il doit être là sur son lieu de travail, puisqu'il fait de l'élevage: investissement : 1120000 A.F. sur plan de la CANA, en faisant un travail de dingue pour mettre en place les équipements, pendant un an et demi. Si ce gars ne doit pas être à «Paysans travailleurs", parce qu'il a un gros investissement, on est fous.

80 Alors que la moyenne de production de poulets fermiers, dans ma région, était de 50 poulets par producteur et par an il y a quarante ans, moi qui fait 240000 poulets par an aujourd'hui, je ne vais pas me détruire à la mitraillette. Dingue... On peut aboutir à des aberrations. La moyenne de lait par vache laitière, il y a 30 ou 40 ans, était de 1300 à 1500 litres de lait par an. C'est-à-dire que pour rester à ce niveau, il faudrait des paysans avec une vache laitière pour rester le " paysan pauvre » type.

81 C'était une explication qui faisait que les gens refusaient de se battre là où l'oppression et l'exploitation capitalistes pesaient sur eux, et passaient leur temps à essayer de découvrir les petits - qui n'y comprenaient rien d'ailleurs, parce qu'on venait à leur 
secours. C'est une attitude cléricale vis-à-vis des petits qui étaient en voie d'élimination. C'est un premier point de contradiction énorme qui a été vécu dans le mouvement et à peu près résolu, notamment dans les dernières journées nationales.

Deuxième type de contradiction : on avait des copains - et on a toujours des copains qui s'installent d'une manière extrêmement économe pour d'autres types de production, à qui on reprochait d'échapper à la loi commune. Non consciemment, implicitement, ceux-là même reprochaient aux autres paysans - la majorité des paysans de l'organisation des « Paysans travailleurs »- de ne pas avoir fait les mêmes choix qu'eux. C'était les gars, par exemple, qui faisaient du fromage de chèvre. On ne voit pas pourquoi on irait les condamner à travailler avec moins de dépenses, moins de productivisme, moins de revenus. Il peut y avoir diverses voies, et cela me parait souhaitable.

83 N.M. : Comment « Paysans travailleurs » a-t-il intégré les problèmes soulevés par les écologistes?

84 Sur le problème de fond qu'on a rencontré autour des questions posées par les écologistes - qui sont parfois mal posées, mais qui représentent de réelles questions on a, nous, vraiment fait un pas que j'estime fondamental, même s'il ne fait qu'ouvrir des voies. Je rappelle les chiffres rapidement: pour l'Europe, en 1979, 95 millions d'hectolitres de lait, qui servaient à faire l'aliment veau-lait, qui permettent défaire au départ 458000 tonnes de beurre, puisque le lait est d'abord écrémé, et qu'ensuite on lui réincorpore des matières grasses. L'Europe en 1979 avait donc exporté 458000 tonnes de beurre, coûtant 5 milliards de francs de subventions à l'exportation. La poudre de lait qui restait était encore trop chère pour donner aux veaux. Elle aurait quand même coûté à peu près aussi cher que du lait entier. On octroie donc encore 2700 milliards de subventions : la prime à la dénaturation. Soit au total 8400 milliards pour produire des animaux qui ont besoin d'hormones pour être rentables, grandir dans de bonnes conditions, et pour produire un aliment qui engendre de façon cyclique des problèmes qui ne sont jamais résolus totalement, notamment les suifs aigris, rancis...

Il y a quantité de problèmes dans les élevages de veaux. On disait, nous, il faut baisser le litre de lait entier de 40 centimes, ce qui coûterait 4 milliards, en supposant qu'on vende la viande de veau faite au lait entier au même prix, soit une économie de 4 milliards sur un budget dont tout le monde dit que c'est un gouffre. Il n'y aurait plus d'excédents de beurre, il en manquerait, on ferait du veau de bonne qualité. La lait entier de la vache, c'est ça qu'il aime, le veau. Je ne sais pas si vous avez remarqué, il y a longtemps, même sans avoir fait de référendum, ni de sondage, c'est l'aliment le plus performant. Il n'y aurait pas de problèmes techniques, même dans un élevage industriel. Actuellement, les marchands d'aliments font monter des silos de 25 à 30 tonnes, qui coûtent 8 millions, chez le paysan qui assure ainsi le stockage. La mise en place de tanks pour conserver le volume de lait nécessaire coûterait moins cher, environ 60000 balles par veau (600 frs), pour garder la viande à 2000 frs chez le producteur ( $20 \mathrm{frs})$. Cette mesure serait intéressante pour l'élevage sous la mère comme pour l'élevage industriel. Il n'y a pas nécessairement d'antagonisme entre ceux que le capitalisme exploite et ceux qu'il élimine, les régions traditionnelles et les régions de production industrielle. Bien sûr, il faudrait réfléchir sur la taille des futurs ateliers et leur implantation régionale. Faudrait-il suivre la concentration laitière de l'Ouest? Il faudrait aussi planifier la production. Mais là on a remis en cause le 
productivisme. On est persuadés que dans d'autres secteurs de production les mêmes problèmes se posent.

De plus, il ne faut pas oublier que pour faire une tonne de poudre de lait, il faut une tonne d'équivalent pétrole (même si mes chiffres doivent être vérifiés) et cela fait pas mal d'Amoco Cadix. Quand vous avez enlevé la crème, vous avez $10 \%$ de poudre de lait qui reste et $90 \%$ de flotte à enlever dans une tour. Dans notre projet, évidemment les tours il faut les mettre dans la "parafine » et bien sûr on nous disait : «Et l'emploi ?» En fait, il faut un gars pour faire marcher la tour, deux au maximum, alors que le simple développement de la consommation de veaux sous la mère, cela maintiendrait beaucoup plus d'emplois en aval.

Premier problème : moi je vois la manière dont on nous fait faire les poulets. On vous produit quelque chose qui est bien plus horrible que le veau aux hormones, ce sont les poulets pour l'exportation. Pour les pays du Moyen-Orient, et pour l'Egypte - parce que l'Arabie saoudite paie pour que Sadate ne soit pas foutu en l'air par des gens qui crèvent de faim dans la vallée du Nil. Les trois exportateurs vont passer à 12 millions de poulets par semaine, perspective 1986. Soit plus de la moitié du marché français de la volaille toutes catégories. Comment le poulet pour l'exportation est-il fait? Dans une telle concentration que le microbisme est fantastique, il faut en permanence des antibiotiques dans les aliments. Je ne parle pas de ceux qu'on ajoute dans l'eau quand il $\mathrm{y}$ a des pépins - mais il n'y a pratiquement plus de bandes où il n'y ait pas de pépins - à cause de la fragilité de ces bêtes-là. Pour que la croissance soit rapide, autrefois on mettait beaucoup de farine de poisson dans les aliments. L'inconvénient, c'est que le poulet avait le goût de poisson, mais ils ont trouvé la formule - faut pas croire, cela ne fait que croître et embellir. Ils y mettent, en plus du soja, 12 à $15 \%$ de graisse. Tout cela est logique, les vaches qui sont de plus en plus sélectionnées ont un manteau de graisse sous la peau ( 50 à $70 \mathrm{kgs}$ de suif par bête), immangeable, à foutre en l'air; plus de la farine de viande - c'est ce qui sort de l'équarrissage. Là aussi, c'est très logique. L'industrialisation fait que les équarrissages ont de plus en plus de cadavres à enlever, intensification, les affaires tournent. Les tuyaux de plastique qui descendent de mes chaînes dans les gamelles, qui sont complètement lisses à l'intérieur, cela se prend dans la masse, il faut secouer, cela ne veut plus descendre, ça pue... Quant aux poulets, cela vous donne deux belles boules de graisse au cul, immangeables. Est-ce qu'on fait de la viande ou de la graisse? On les tue à la sortie de l'enfance, ils ont le goût de papier mâché. Après, ils foutent ces poulets là à refroidir pour les congeler dans de l'eau maintenue à $2^{\circ}$, et ils y restent pendant 4 heures. Les poulets absorbent alors $15 \%$ de leur poids en eau. Cela vous fait de bons poulets en sauce, enveloppés dans une matière plastique dont les cellules sont migrantes - le polystyrène. Il y a des cellules de plastiques qui rentrent dans la viande, c'est vachement grave et cancérigène ("Que Choisir » en parle d'ailleurs). Je ne veux pas vous effrayer, mais ce que je peux vous dire, c'est que si vous mangez un poulet par semaine,comme les antibiotiques doivent être changés tous les deux mois, vous êtes à peu près sûr que quand vous arriverez à l'hôpital vous serez tranquille, les antibiotiques ne vous feront rien, il y aura accoutumance, le spectre est assez large.

Ecoutez, je sais bien qu'on ne peut pas revenir en arrière, au poulet de grand-mère, c'est vrai que cela ne correspondrait pas aux besoins. Mais je sais que les oiseaux, comme les volailles, ont un broyeur qui s'appelle le gésier, ils mangent des petits cailloux qui se placent dans le gésier et servent à broyer des graines. C'est des graines 
qu'ils manger. Si bien que l'aliment étant broyé, les industriels mettent de l'argile dedans pour refaire des graines. Et pourtant les oiseaux et les volailles, cela vient très bien avec des céréales, mais cela met plus de temps.

Il y a moyen de faire, industriellement, avec moitié moins de poulets au mètre carré, et avec un tiers de temps de fabrication en plus, c'est-à-dire avec quatre producteurs là où il y en a un aujourd'hui. Mais on fera en plus des économies d'énergie, avec un autre type d'alimentation, avec une remise en cause de la production. On n'a pas fait d'études sur ce thème, on a été les premiers pour l'affaire du veau, mais depuis que l'INRA est en train de destiner ses recherches aux entreprises agro-alimentaires, aucun copain de l'INRA ne fait plus ce type d'analyse...

En faisant une étude qui prendrait en compte tous les éléments, je ne suis pas sûr que ce soit même valable sur le plan économique, défaire le poulet comme je le fais. Si je change et que je fais du poulet fermier; qu'est-ce que je vais en faire, de mes poulets? Il sera bien plus cher, il faudra que je le porte en ville, que je le tue... Je le vendrai dans le $\mathrm{xvI}^{\mathrm{e}}$. On peut prendre le porc, on peut prendre les légumes, et les résidus d'azote dans les salades. Ou encore l'expérience faite avec des tomates produites à coup d'azote et d'eau en pagaille : elles étaient destinées à faire de la sauce tomate, l'intensification faisant gagner $20 \%$ de rendement; mais il a fallu les réduire de $20 \%$ de plus que les autres tomates pour faire de la sauce aussi concentrée. Cela fait quand même des échanges de vapeur. On devient fou, comment faire?

91 Nous, on pense qu'il faut refaire une toute autre politique agricole. A quel prix est-ce que tout cela peut être accessible aux paysans? Il faut qu'une remise en cause du productivisme puisse être généralisée, que la politique agricole soit déterminée de manière contractuelle, voire tripartite puisque que les Pouvoirs Publics contrôlent la banque, le Crédit Agricole, les droits d'accès aux prêts des paysans. Le prêt est l'élément qui permet d'avoir l'outil de travail qui nous permet d'exercer notre profession. Un droit qui devrait être associé à des volumes limités de productions réalisées avec certaines techniques.

92 Il faut répartir le travail autrement. Il y aura peut-être trois à quatre paysans de plus à faire du poulet ; il faut que ce travail soit payé, tous frais déduits, étant entendu qu'on ne pleurera pas si les bâtiments ne nous appartiennent plus. Notez bien qu'on ne va pas dans ce sens là : DOUX, ${ }^{29}$ pour qu'on reste propriétaire de nos bâtiments et pour ne pas nous payer plus cher, subventionne l'installation d'un bâtiment à fonds perdu, pour des sommes variant entre trois et six millions par bâtiment. Et cela pour nous laisser la propriété théorique, parce que c'est comme cela qu'ils nous exploitent le mieux : des petits planteurs de cacahuètes entre les mains de la grosse boîte. Restez petits planteurs, toute la famille travaillera gratuitement. C'est la Banque Mondiale qui écrit cela dans un rapport de Mac Namara, à propos du Tiers Monde.

Dans cette perspective, il faut obtenir le paiement du travail, pour une quantité de travail déterminée, et des produits d'une qualité dont on débat publiquement, y compris avec les organisations de consommateurs. En d'autres termes, nous voulons avoir des conventions collectives à deux niveaux : pour accéder au métier, et pour vivre mieux. On fera un progrès fantastique lorsqu'on en aura. On est avant 1936, nous, il y a encore du boulot à faire, pratico-pratique. Il y a un an, je n'aurais pas pu vous tenir ce langage. Bien sûr, on théorise quand on agit, et on a commencé par agir. C'est, je crois, le propre du monde du travail. Cela ne nous empêche pas d'avoir la capacité intellectuelle de théoriser, de comprendre. 
94 Avec l'affaire du veau aux hormones, on avait les éléments du dossier au mois de décembre 1979, et on a déposé la plainte à la fin août, et tout ce temps a été un moment de débat intense interne au mouvement. On s'est posé de redoutables questions. Ce n'est pas simple d'encourir la menace de la ruine de milliers d'éleveurs de veaux. Cela ne s'est heureusement pas si mal passé.

Il faut dire qu'avec l'U.F.C, on s'est un peu engueulé. On n'est pas contre un boycott, mais quand on pose le principe d'un boycott, il nous semble nécessaire en même temps de poser le problème de la garantie des travailleurs, les garanties de survie. On ne peut pas jouer les irresponsables politiques, on doit exiger qu'il ne soit pas porté atteinte à la vie économique des travailleurs.

En fait, l'U.F.C. nous a coiffés, parce qu'ils savaient qu'on hésitait. Elle a décidé le boycott la veille de la conférence de presse qui était prévue en commun. Le langage de l'U.F.C. était : "Si vous nous donnez un label sur le veau, on enlèvera le boycott ». Or, avec la pratique du label dans le domaine du poulet, de la pintade, de tout ce que tu voudras, pour un revenu qui reste le même, le producteur a environ un tiers de charges en plus (temps de production allongé, meilleure nourriture) pour un même revenu net. Et dans le même temps, les prix augmentent à la consommation et les intermédiaires en prennent une bonne partie. Cela revient à dire au gars de banlieue : «Tu n'en mangeras pas, ou tu mangeras de la merde ». C'est clair, non? J'ai été sec avec l'U.F.C, en plus il y avait Brice Lalonde. Lalonde nous a suivi pendant toute cette période, y compris quand on est allé foutre le bordel à Strasbourg, au Parlement Européen, il était passionné, mais il a tout le temps maintenu, jusqu'au bout, malgré toutes nos explications, le thème du label.

97 Les gars ont donc été très présents dans la décision et les éleveurs de veau ont vraiment été au centre de la décision, principalement ceux qui avaient contribué à créer une organisation syndicale face à la coopérative d'Ancenis et face à Sanders en Vendée et en Loire-Atlantique: Jean Guilbert, Jean-Marie Guérineau... qui étaient des gens qui étaient peu apparus dans le mouvement, se sont révélés des types maîtrisant vraiment bien les problèmes.

On cherche, on réfléchit, je n'ai pas tout prêt. Si je savais, François (Guillaume) serait venu me voir pour la politique agricole qu'il faut mener. Quel soulagement si on avait l'accès automatique aux prêts! Vous savez comment cela se passe? Le Crédit est mutuel, c'est l'affaire bancaire la plus démocratique. Alors vous allez voir ce qui la distingue des autres banques :

99 1. Quand vous allez faire du crédit à la consommation dans un supermarché, il n'y a pas de mouchards dans les HLM. Le Crédit Agricole, lui, a un mouchard dans chaque commune, qui est administrateur de la Caisse Locale et qui est là uniquement, non pas pour calculer les taux d'intérêt ni la répartition des excédents - c'est fixé ailleurs mais pour dire : Il pourra rembourser ou il ne pourra pas rembourser.

100 2. Quand un gars va discuter son dossier, soit pour un rattrapage de financement, soit pour s'installer, il est rigoureusement interdit sur tout le territoire français qu'il se fasse accompagner d'un tiers et encore moins d'un représentant syndical. Nous, on tourne la difficulté. Le gars y va et demande des suspensions de séances. La dernière fois, au bout de quatorze suspensions, le directeur adjoint est descendu pour me causer. Cela durait depuis le matin. 
101 3. Il y a un contrat de prêt qui est fait, en quatre ou huit pages, pour le contrat d'assurance invalidité/décès...Je vous affirme qu'avant d'apposer le «Lu et approuvé », il n'y a pas $1 \%$ des gars qui le lisent. Et en particulier qui lisent une clause stipulant pour un individu que, s'il oublie de rembourser une annuité d'un seul prêt, la banque peut lui exiger immédiatement toutes les annuités de tous les prêts. C'est prodigieux.

102 4. Quand il y a un dossier difficile, croyez-vous que les administrateurs de la Caisse locale en discutent? Non, il vient un inspecteur de la Caisse départementale qui fait l'étude économique. Je préfère avoir affaire à un inspecteur de la PJ.. Je le dis, parce qu'un inspecteur de la PJ., par mon avocat, je peux avoir son rapport. Le gars à qui on vient pomper tous ses chiffres, pour en sortir un bilan, compte d'exploitation, budget prévisionnel, qu'a-t-il foutu là-dedans pour pouvoir éventuellement les contester? D'autant plus que, pris à froid, il en oublie toujours, des chiffres, des avances aux cultures. Il y a toujours interdiction de donner le double des rapports de l'inspecteur, interdiction de donner les doubles des contrats de prêts. On est démocratique, on est mutuel ou on ne l'est pas... J'aime mieux la P.J.

103 Le Crédit Agricole profite du fait que les mecs ont honte d'avoir des dettes. Dans ma région, l'esprit chrétien n'y a pas été pour rien. L'Eglise catholique, c'est l'alliance du goupillon et de l'argent. Le résultat, c'est que les gars s'appuient sur la famille pour cacher, éponger la dette, sur les beaux-frères, les belles-mères, les pères, les frères et soeurs qui vendent un bout de terrain, qui s'endettent aussi. Le Crédit Agricole récupère toujours ses billes. Voilà la banque dans laquelle on est.

104 N.M. : En tant que dinosaure, comment vois-tu le rôle des « anciens » dans « Paysans travailleurs »?

105 B.L. : Vis-à-vis des anciens, de ceux qui portent un peu la mémoire collective, il n'y a plus ce phénomène de rejet, qui a été fort pendant toute une période. Je le sais bien, parce que j'ai fait partie de ceux-là. La technique est devenue simple. On a quelques gars qui ont un certain potentiel, quand le collectif national estime qu'il a besoin d'eux, il les prend là, il les met là, et après il leur dit, tu rentres. Moi, par exemple, on me dit "Tu iras à une conférence Agenor à Bruxelles ", je vais pendant deux jours à Bruxelles, et puis on refait le monde au niveau européen.

106 F.B.: Sébastien COX est parti mais je pense qu'il voulait te reposer le problème quotaslquantums par rapport au Tiers Monde. Ce n'est pas évident de répondre.

107 B.L. : Non, car je ne m'y connais pas assez. Dieu sait si on essaie pourtant de bosser làdessus. C'est encore trop lié à des préoccupations de revenu des gars ici, mais on croit savoir quelles sont les conséquences logiques d'un certain développement impérialiste, de nouvelles répartitions internationales de travail qui ont lieu dans le secteur industriel, mais aussi dans le secteur alimentaire. La répartition internationale concernant la viande semble avoir des conséquences concrètes en Amérique du Sud, plus spécialement en Argentine, pas seulement pour ce secteur. Vis-à-vis des productions légumières, il semble qu'une nouvelle répartition internationale du travail semble s'effectuer sur le pourtour de la Méditerranée et bien au-delà des futurs pays qui doivent entrer dans le Marché Commun: dans la vallée du Nil, en Turquie, au Sénégal. On se pose d'énormes questions quand, par exemple au Sénégal, dans cette région proche du Sahel, on remplace les cultures vivrières par des cultures de haricots verts importés en contre-saison, et même maintenant en pleine saison puisque l'été dernier cela arrivait à 7 francs le kilo à Orly. Est-ce qu'on ne les fait pas crever en nous 
faisant crever? Je ne veux pas prendre position, mais il me semble que c'est une volonté de pillage, de développement de l'échange inégal et qu'en exploitant à mort les gens de ces pays, on nous tue tous. On a fait des journées à Nantes sur le problème des paysans du Tiers Monde, un peu sur ces thèmes-là. Nous, on dit cela, et la FNSEA prépare sa participation à la constitution de l'arme de demain, l'arme alimentaire...

J'ai parlé un peu beaucoup, je m'en excuse, on ne se voit pas si souvent. Bon appétit, j'espère qu'il y aura du poulet...

\section{BIBLIOGRAPHIE}

F. BOURQUELOT , N. MATHIEU, 1987, « Aux origines des ruptures paysannes : les années 1968 à 1974 », in Les agriculteurs et la politique depuis 1970, Colloque des 30 novembre, 1 et 2 décembre 1987, Association Française de Science Politique, Paris.

R. BOURRIGAUD, 1987, «Origines idéologiques de la nouvelle gauche paysanne : le cas de la Loire-Atlantique ", in Les agriculteurs et la politique..., op. cit.

Y. CHAVAGNE, 1988, Bernard Lambert, Trente ans de combat paysan. Préface de Bernard Thareau, La Digitale/Association Bernard Lambert, Quimperlé.

M. DEBATISSE, 1963, La révolution silencieuse, Calmann-Lévy, Paris.

R. HUBSCHER, R.M. LAGRAVE, 1989, France : une fraternité en lutte », in « Les

organisations syndicales et professionnelles agricoles en Europe ", Cahiers du CEVIPOF, 5, Paris.

R.M. LAGRAVE, 1987, « La gauche paysanne : entre dissidence et concurrence (MODEF,

CNSTP, FNSP) », in Les agriculteurs et la politique....op. cit.

B. LAMBERT, 1970, Les paysans dans la lutte des classes, Le Seuil, Paris.

\section{NOTES}

1. Né en 1931 en Loire-Atlantique, Bernard Lambert a été Président du C.N.J.A. de ce département (1956), responsable national et membre du bureau national de la J.A.C. (1964), Président de la S.I.C.A. et de la S.A.V.A. de Challans (1965). De 1964 à 1970, il est Secrétaire Général de la F.D.S.E.A. et de la F.R.S.E.A. de l'Ouest. Député M.R.P. de la Loire-Atlantique de 1958 à 1962, il adhère au P.S.U. en décembre 1966 et devient, l'année suivante, membre du Comité National de ce Parti.

2. Voir photo. Nous remercions l'Association Bernard Lambert de nous l'avoir aimablement communiquée. Félicitations pour tout le travail qu'elle fait pour la mémoire de l'histoire paysanne.

3. Cf. mots en caractères gras. «On a identifié et analysé quatre formes principales de rupture : une critique radicale de la pratique syndicale analogue à celle qui vise le « mandarinat », qui a entraîné un renouvellement des formes d'action politique et des 
pratiques de luttes ; la remise en cause des positions sur le « pouvoir économique » qui se concrétisa par une éradication de l'idéologie " coopérative "; la radicalisation des critiques sur la politique des structures s'accompagnant d'une rupture idéologique fondamentale sur le rapport à la terre et à la propriété ; un renouvellement de la réflexion sur les positions de classe des couches paysannes par rapport aux autres catégories de travailleurs et dans la relation avec l'industrie et le capitalisme (luttes autour du thème de l'intégration). Dans tous les cas, pour la première fois, la lutte et le " concret » ont été considérés comme des moyens de définir les propositions à adopter, qu'elles soient idéologiques ou politiques. » F. BOURQUELOT et N. MATHIEU, 1987. 4. COFNA (Confédération des Fabricants pour Animaux) siège social à Tours, à l'époque filiale d'UNILEVER.

5. Bernard Lambert s'est toujours exprimé en anciens francs.

6. Michel Debatisse, ancien petit agriculteur laitier du Puy-de-Dôme, a été Secrétaire général de la Jeunesse agricole catholique de 1954 à 1957, et du Centre national des Jeunes Agriculteurs de 1958 à 1964. Président de la F.N.S.E.A. de 1971 à 1979, il a été nommé secrétaire d'Etat auprès du Premier ministre, chargé des industries agroalimentaires, de 1979 à 1981. Représentant à l'Assemblée des communautés européennes depuis 1979, il est aussi Conseiller régional d'Auvergne.

7. La loi de 1964 prolonge la loi d'orientation de 1960 ; elle porte sur l'organisation contractuelle et les interprofessions. Elle propose aux partenaires de l'échange (agriculteurs, industriels et commerces) des principes et des modalités d'un régime contractuel en agriculture.

8. F.N.S.E.A. : Fédération Nationale des Syndicats Agricoles, créée en 1945 au sein de la Confédération générale de l'Agriculture (C.G.A.).

9. F.R.S.E.A. : Fédération régionale des syndicats d'exploitants agricoles, la seule fédération régionale. Bernard Lambert a été secrétaire général de la Fédération départementale et de la Fédération régionale de 1964 à 1970.

10. Sicco Mansholt, Commissaire européen (responsable du secteur agricole au sein de la Commission européenne) a établi en 1968 un mémorandum (dit plan Mansholt) préconisant une diminution de la population active agricole, une diminution de la S.A.U., une réforme des structures, et la création d'unités de dimensions plus importantes. En 1969, en France, le rapport Vedel, établi par une commission d'experts gouvernementaux animée par le doyen Vedel, eut un grand retentissement. Il préconisait de repenser la politique agricole française et de pousser à une restructuration plus efficace des exploitations agricoles.

11. Bernard Lambert a adhéré au Parti socialiste unifié (PSU) en décembre 1966. Il avait été député M.R.P. de la Loire-Atlantique (1958-1962).

12. C.N.J.A. : Centre national des Jeunes Agriculteurs, syndicat créé en 1956, issu du Cercle des Jeunes Agriculteurs qui était au sein de la F.N.S.E.A à sa création en 1945. 13. Bernard Thareau, militant du C.D.J.A de Loire-Atlantique et de l'Ouest (C.R.J.A.O.), actuellement responsable des questions agricoles au PS.

14. Une agriculture au service des travailleurs, in Frères du Monde, $n^{\circ} 54-55,4 / 5,1968$, p. 2-77.

15. M.R.J.C : Mouvement rural de la Jeunesse chrétienne.

16. II s'agit de la C.A.N.A, Coopérative agricole la Noëlle d'Ancenis.

17. B. LAMBERT, 1970, Les paysans dans la lutte des classes, Paris, Seuil.

18. M.O.D.E.F. : Mouvement de coordination et de défense des exploitants familiaux, créé en 1959 et lié au P.C. 
19. Née en 1970, « Paysans travailleurs » est une tendance jusqu'à son institutionalisation en 1974 en Association nationale des paysans travailleurs (A.N.P.T) ; elle ne prendra le statut syndical qu'en 1981, en regroupant plusieurs tendances et syndicats minoritaires dans la C.N.S.T.P. (Confédération nationale des syndicats de travailleurs paysans).

20. F.G.A. : Fédération générale de l'agriculture (C.F.D.T.), syndicat de salariés de l'agriculture et de l'agro-alimentaire.

21. Grand propriétaire de l'Aube ou de la Champagne et ancien président de l'A.G.P.B. (Association générale des producteurs de blé).

22. Le 16 septembre 1977, la C.E.E établit une taxe parafiscale de 1,5\%, dite de coresponsabilité, sur le lait. Elle était retenue par les coopératives et firmes sur le prix payé au producteur ; en 1981, cette taxe est portée de 2 à 2,5\%.

23. M.S.T.T. : Mouvement syndical des travailleurs de la terre.

24. U.F.C. : Union fédérale des consommateurs

25. C.S.F. : Confédération syndicale des familles

26. 23 C.S.C.V : Confédération syndicale du cadre de vie.

27. Allusion aux luttes foncières (terre reprise aux fermiers par un propriétaire ou achat par un gros propriétaire cumulard).

28. Cf. Enquête à la campagne de Mao-Tsé-Toung, à laquelle se référaient divers groupes maoïstes de 1970 à 1974 .

29. Etablissements DOUX : intégrateur de producteurs de volailles.

\section{RÉSUMÉS}

Le texte publié est la transcription des paroles prononcées par Bernard Lambert le 4 mai 1981 au séminaire «Luttes dans et pour l'espace rural» de Françoise Bourquelot et Nicole Mathieu à l'EHESS. Un récit de trente ans d'histoire du syndicalisme paysan, qui montre une pensée d'avant-garde, une ouverture sur les problèmes internationaux, une capacité de se projeter dans l'avenir.

A former union member testimony and commitment

The history of French farmers' unions since thirty years is related by Bernard LAMBERT (1931-1984), a long-time trade unionist, founder member of the union « Paysans travailleurs » and author of the book entitled Les paysans dans la lutte des classes (1970).

Bernard LAMBERT's talk to a seminar held in Paris on May 4th 1981 at the EHESS, reveals, through debates put back in their context, his avant-garde ideas, his concern for international problems and his will to anticipate the future.

INDEX

Keywords : France, Bretagne, agriculture, farmers\&\#39, union, trade unionism, biography, social history

Mots-clés : syndicalisme paysan, biographie, « Paysan travailleur », Loire-Atlantique 


\section{AUTEURS}

\section{BERNARD LAMBERT}

Bernard LAMBERT (1931 - 1984), qui fut l'un des fondateurs du mouvement « Paysans travailleurs ", a marqué le syndicalisme paysan par sa constante capacité de contestation et de rupture. Il a publié en 1970 : Les paysans dans la lutte de classes.

\section{FRANÇOISE BOURQUELOT}

Maître de conférences à l'EHESS, elle a connu Bernard Lambert dans sa jeunesse, lorsqu'elle était elle-même responsable nationale de la branche « adolescentes » de la J.A.C. Elle est connue pour ses travaux sur les salariés agricoles, qu'elle poursuit actuellement en étudiant les maraîchers-serres du Languedoc-Roussillon. Mais le lien entre ses recherches et le milieu militant dont elle est issue n'a jamais été rompu, de même que son amitié avec Bernard Lambert.

\section{NICOLE MATHIEU}

Directrice du laboratoire STRATES, directeur de recherches au CNRS, responsable de l'axe « Evolution des exploitations agricoles dans leur environnement régional » dans le G.D.R. AGRAL, elle a connu l'amitié de Bernard Lambert et d'autres paysans révolutionnaires encore en vie. 